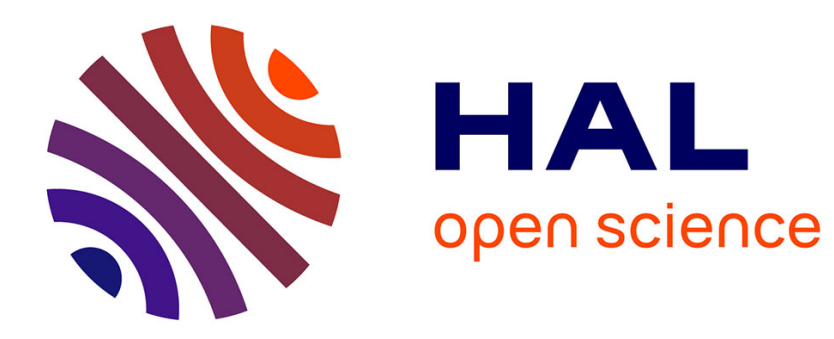

\title{
Le poids spécifique des feuilles: un indice de production de quatre graminées fourragères
}

Basile Noïtsakis

\section{To cite this version:}

Basile Noïtsakis. Le poids spécifique des feuilles: un indice de production de quatre graminées fourragères. Agronomie, 1986, 6 (1), pp.115-117. hal-00884856

\section{HAL Id: hal-00884856 https://hal.science/hal-00884856}

Submitted on 1 Jan 1986

HAL is a multi-disciplinary open access archive for the deposit and dissemination of scientific research documents, whether they are published or not. The documents may come from teaching and research institutions in France or abroad, or from public or private research centers.
L'archive ouverte pluridisciplinaire HAL, est destinée au dépôt et à la diffusion de documents scientifiques de niveau recherche, publiés ou non, émanant des établissements d'enseignement et de recherche français ou étrangers, des laboratoires publics ou privés. 


\title{
Le poids spécifique des feuilles : un indice de production de quatre graminées fourragères
}

Basile NOÏTSAKIS

Laboratoire de Science des Pâturages (236), Université de Thessalonique, 54006 Thessalonique, Grèce

RÉSUMÉ

\begin{abstract}
Le poids spécifique des feuilles, c'est-à-dire le poids de feuille par unité de surface foliaire, ainsi que la variation de matière sèche des feuilles à différents stades phénologiques ont été étudiés chez quatre graminées fourragères : Phalaris tuberosa var. stenophylla, Phataris tuberosa, Dactylis glomerata cv. " Palaistina " et Dactylis glomerata cv. "Prairial ». La plus grande valeur du poids spécifique observée chez $P$. $t$. var. stenophvlla peut être attribuée au poids des feuilles plus important. Le poids spécifique peut être utilisé comme un indice de production fourragère.
\end{abstract}

Mots clés additionnels : Surface foliaire, poids de feuille, production fourragère.

Specific leaf weight : an index of dry matter production.

The specific leaf weight, as well as changes in leaf dry matter, of Phalaris tuberosa var. stenophylla, Phalaris tuberosa, Dactylis glomerata cv. "Palaistina", Dactylis glomerata cv. "Prairial" were examined at different phenological stages. The highest specific leaf weight was found in $P$. tub. var. stenophylla and may be attributed to the highest leaf weight. Specific leaf weight can be used as an index of forage production.

Additional key words : Leaf area, leaf weight, forage production.

\section{INTRODUCTION}

L'amélioration quantitative des pâturages a pour base la productivité d'espèces fourragères. Plusieurs études concernant l'analyse quantitative de croissance, ainsi que ses relations avec l'activité photosynthétique des plantes ont été réalisées (CHATTERTON et al., 1972 ; FREY \& MOSS, 1976 ; BLANCHET \& GELFI, 1978 ; SCOTT \& BATCHELOR, 1979 ; SHIH \& GASCHO, 1980). Il serait donc intéressant de proposer des indices de production fourragère faciles à mesurer et à exprimer.

Ce travail analyse la variation du poids de feuilles par unité de surface foliaire ainsi que la variation de la production de matière sèche totale chez 4 graminées fourragères: Phalaris tuberosa, Phalaris tuberosa var. stenophylla, Dactylis glomerata cv. "Prairial", Dactylis glomerata cv. «Palaistina » en fonction des stades de croissance.

Le poids de feuilles par unité de surface foliaire (poids spécifique des feuilles) peut être un indice, utile à l'amélioration des pâturages, de la production fourragère chez ces 4 graminées.

\section{MATÉRIEL ET MÉTHODES}

La station expérimentale où les mesures ont été effectuées est caractérisée par un climat semi-aride avec une hauteur moyenne des pluies de $430 \mathrm{~mm}$ et une température moyenne de $15,5^{\circ} \mathrm{C}$. Il faut signaler, que, au cours de la période de croissance des plantes, 2 déficits hydriques d'une durée totale de $38 \mathrm{j}$ (fig. 1) sont apparues dans le sol.

En 1976, 950 plantes de chaque espèce, semées en pots, ont été repiquées dans les parcelles de dimensions $2,8 \times 1,0 \mathrm{~m}$ (densité : 340 plantes $/ \mathrm{m}^{2}$ ). Le protocole expérimental a été le suivant : sur 4 placettes de $9 \mathrm{dm}^{2}$, on a coupé 4 fois la biomasse aérienne au cours de la période de croissance $(30 / 3,30 / 4,17 / 5$, $15 / 6)$. Il y a eu 4 répétitions aléatoirement réparties dans chaque parcelle. 


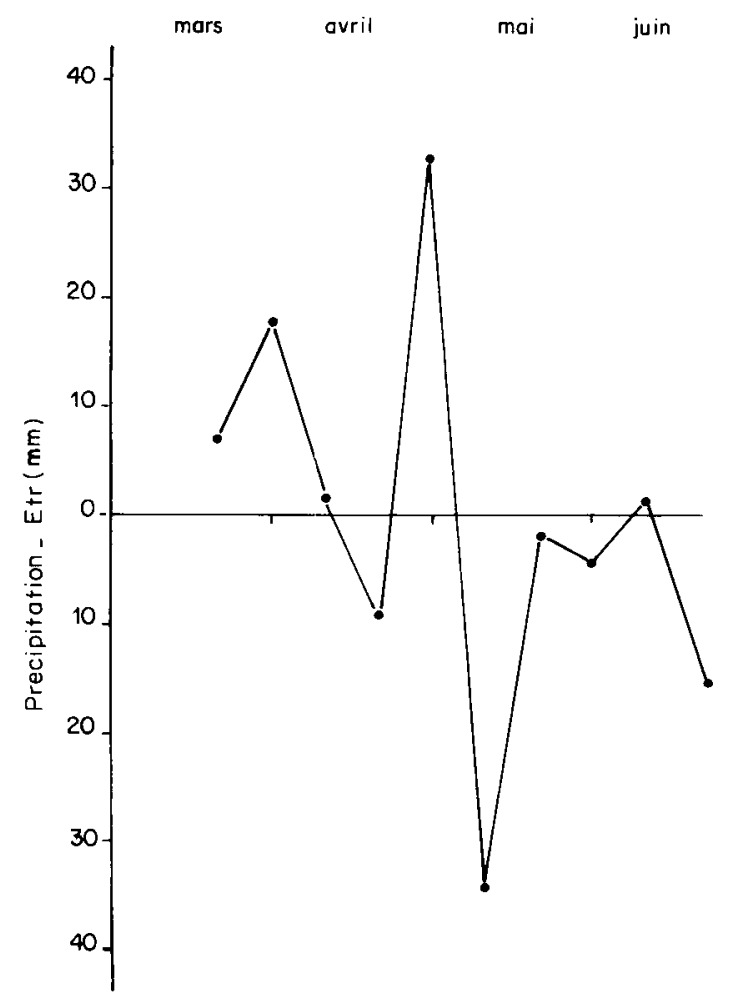

Figure 1

Evolution, au cours du printemps de 1982, de la différence: Précipitation-ETR $(\mathrm{mm})$ à la station expérimentale.

Difference (mm) between precipitation and ETR at the experimental station over spring 1982.

On a mesuré la surface foliaire d'un souséchantillon de 20 feuilles à l'aide d'un planimètre électronique. On a évalué également le nombre de feuilles présentes dans chaque échantillon et, en même temps, on a mesuré son poids sec.

On a ainsi pu facilement calculer le poids spécifique des feuilles à partir du rapport : poids sec/surface foliaire.

\section{RÉSULTATS ET DISCUSSION}

On peut observer (fig. 2) que la variation saisonnière du poids spécifique est faible et surtout qu'à partir de la mi-avril la valeur du poids spécifique est relativement stable. Une stabilité semblable a été observée par Evans (1972) chez Helianthus annuus, par AASE (1978) chez le blé, par FREY \& MOSS (1976) chez Hordeum vulgare.

Par contre, Evans \& Hughes (1961) ont trouvé une réelle sensibilité du poids spécifique aux changements climatiques chez Impatiens parviflora. Le désaccord observé pourrait s'expliquer par des conditions expérimentales particulières, permettant seulement à $67,42,25$ et 7 p. 100 du rayonnement solaire d'arriver aux plantes, et par un hypothétique comportement particulier d'I. parviflora.

Cette stabilité du poids spécifique des feuilles se traduit par une relation linéaire entre la surface foliaire et le poids de M.S. des feuilles. La corrélation varie entre $r^{2}=0,978$ et $r^{2}=0,998$ selon l'espèce, la

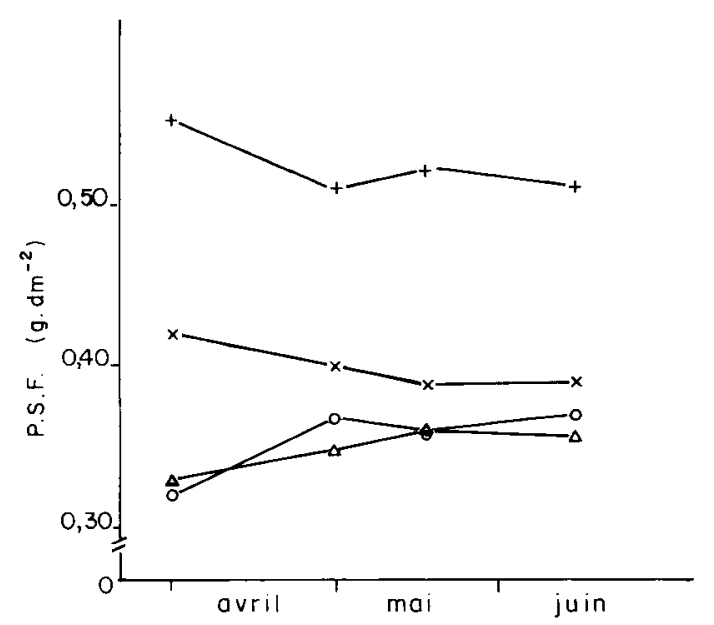

Figure 2

Evolution du poids spécifique des feuilles chez Phalaris tuberosa var. stenophylia $(+)$, P. tuberosa $(\times)$, Dactylis glomerata $c v$. «Palaistina» $(\triangle)$ et D. glomerata $c v$. «Prairial » $(\bigcirc)$.

Specific leaf weight in Phalaris tuberosa var. stenophylla $(+)$, P. tuberosa $(\times)$, Dactylis glomerata $c v$. Palaistina $(\triangle)$ and D. glomerata $c v$. Prairial (O).

valeur limite étant de 0,980, 2 degrés de liberté, au seuil $\mathrm{P}=0,01$.

La figure 2 montre que la valeur du poids spécifique chez $P$. $t$. var. stenophylla est sensiblement plus élevée que celle de $P$. tuberosa, cette dernière étant plus élevée que celle des 2 cultivars de dactyle. La plus grande valeur du poids spécifique des feuilles chez $P$. $t$. var. stenophylla peut être attribuée au poids plus important de feuilles; la production de matière sèche des feuilles est sensiblement plus élevée que celle des autres graminées.

$\mathrm{Si}$ on admet qu'il existe une corrélation positive entre le poids spécifique des feuilles et l'activité photosynthétique de la partie aérienne (FREY \& MOSS, 1976), on peut dire que $P$. $t$. var. stenophylla présente (fig. 3) une production plus élevée comparée à celle des autres graminées étudiées.

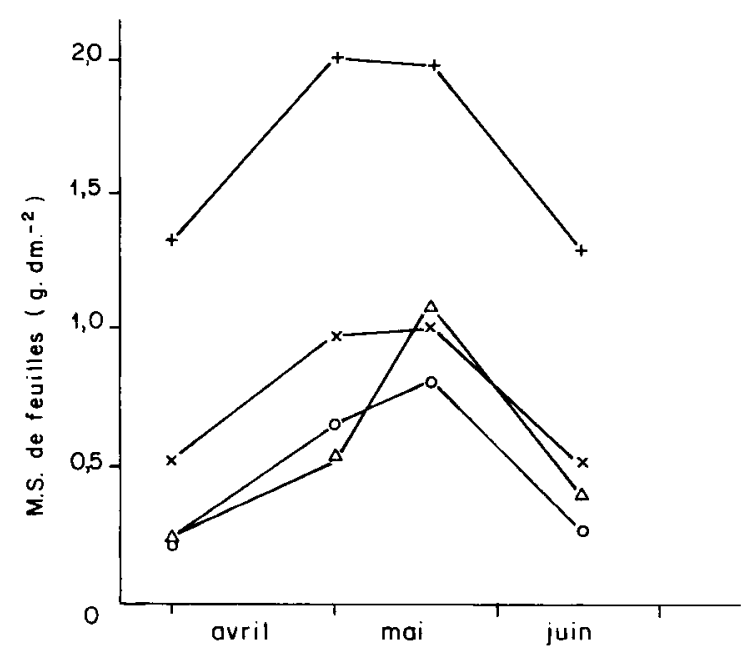

Figure 3

Evolution de la matière sèche des feuilles chez Phalaris tuberosa var. stenophylla $(+)$, P. tuberosa $(\times)$, Dactylis glomerata $c v$. "Palaistina " $(\triangle)$ et D. glomerata $c v$. «Prairial » $(\bigcirc)$.

Leaf dry weight in Phalaris tuberosa var. stenophylla $(+)$, P. tuberosa $(\times)$, Dactylis glomerata $c v$. Palaistina $(\triangle)$ and D. glomerata cv. Prairial $(\bigcirc)$. 


\section{CONCLUSION}

Les résultats obtenus suggèrent que Phalaris tuberosa var. stenophylla, ayant un poids spécifique des feuilles plus élevé par rapport à d'autres espèces, peut être considéré comme une espèce plus productrice. La valeur du poids spécifique comme indice de production pourrait être utilisée à l'amélioration quantitative des pâturages méditerranéens.

Reçu le 12 novembre 1984. Accepté le 11 septembre 1985.

\section{RÉFÉRENCES BIBLIOGRAPHIQUES}

Aase J. K., 1978. Relationships between leaf area and dry matter in winter wheat. Agron. J., 70, 563-565.

Blanchet R., Gelfi N., 1978. Relations entre développement foliaire, transpiration et production chez le soja (cv. Amsoy 71 et Hodgson). Ann. agron., 29, 223-242.

Chatterton N. J., Lee D. R., Hungerford W. F., 1972. Diurnal change in specific leaf weight of Medicago sativa L. and Zea mays L. Crop Sci., 12, 576-578.

Evans G. C., 1972. The quantitative analysis of plant growth. Studies in Ecology. Vol. 1. Blackwell Sci. Publ., Oxford, London, Edinburgh, Melbourne, $733 \mathrm{p}$.
Evans G. C., Hughes A. P., 1961. Plant growth and the aerial environment. I. Effect of artificial shading on Impatiens parviflora. New Phytol., 60, 150-180.

Frey M. N., Moss D. N., 1976. Variation in RuDPCase activity in barley. Crop Sci., 16, 209-213.

Scott H. D., Batchelor J. T., 1979. Dry weight and leaf area production rates of irrigated determinate soyabeans. Agron. J., 71, 776-782.

Shih S. F., Gascho G. J., 1980. Relationship among stalk length, leaf area and dry biomass of sugarcane. Agron. J., 72, 309-313. 